\title{
CT and MR Imaging in the Diagnosis of Scleritis
}

\author{
(D) M.C. Diogo, (D) M.J. Jager, and DT.A. Ferreira
}

\begin{abstract}
SUMMARY: Scleritis is a rare, underdiagnosed vision-threatening condition that can occur isolated or in association with other orbital abnormalities. The etiology of scleritis is mainly inflammatory noninfectious, either idiopathic or in the context of systemic disease. Ultrasonography remains the criterion standard in diagnostic imaging of this condition but might prove insufficient, and studies on the diagnostic value of CT and MR imaging are lacking. We retrospectively analyzed 11 cases of scleritis in which CT and/or MR imaging were performed during the active phase of disease and assessed the diagnostic utility of these techniques. The most important imaging findings of scleritis were scleral enhancement, scleral thickening, and focal periscleral cellulitis. MR imaging is the recommended imaging technique, though posterior scleritis also can be accurately diagnosed on CT. It is important for the radiologist to be acquainted with these findings because being able to diagnose scleritis is of clinical significance and might be vision-saving.
\end{abstract}

ABBREVIATIONS: $C E C T=$ contrast-enhanced $C T$; IOID = idiopathic orbital inflammatory disease

$\mathrm{s}$ cleritis is a rare but often treatable vision-threatening condition that can occur isolated or in association with other orbital abnormalities. ${ }^{1,2}$ The most common etiology is inflammatory (noninfectious, $90 \%$ of patients), either idiopathic in the spectrum of idiopathic orbital inflammatory disease (IOID)/pseudotumor or in the context of a systemic disease. ${ }^{3}$ Infectious scleritis is rare (7\% of cases) and is associated with predisposing factors such as surgery or trauma.,

Although the diagnosis of scleritis is usually based on clinical assessment and ultrasonography, ${ }^{3,5}$ the variable clinical presentation, variable ultrasonography findings, and unfamiliarity with the diagnosis account for posterior scleritis being one of the most underdiagnosed conditions in ophthalmology. ${ }^{6}$

Improvements in CT and MR techniques have led to important progress in ophthalmologic imaging. ${ }^{7}$ However, detailed studies concerning imaging of scleritis, especially MR imaging, are few and contain only small numbers of patients. ${ }^{5,6,8-11}$

This article considers the role of CT and MR imaging in the diagnosis of scleritis, emphasizing the array of imaging findings in 11 cases.

Received December 20, 2015; accepted after revision June 14, 2016.

From the Department of Neuroradiology (M.C.D.), Centro Hospitalar de Lisboa Central, Lisbon, Portugal; and Departments of Ophthalmology (M.J.J.) and Radiology (T.A.F.), Leiden University Medical Center, Leiden, the Netherlands.

Please address correspondence to Mariana Cardoso Diogo, MD, Departamento de Neurorradiologia, Hospital de São José, Rua José António Serrano, 1150 - Lisboa, Portugal; e-mail: mariana.cardoso.diogo@gmail.com

http://dx.doi.org/10.3174/ajnr.A4890

\section{CASE SERIES}

We describe a series of 11 cases of scleritis in 10 different patients. All cases had CT and/or MR imaging performed during the active phase of disease. Scleritis was confirmed by an experienced ophthalmologist by using clinical and imaging criteria and by the therapeutic response in doubtful cases.

CT was performed in 8 cases of unilateral scleritis and MR imaging in 6 unilateral and 1 bilateral case of scleritis.

Table 1 lists the clinical and diagnostic information for the 10 patients who form the basis of this report. All patients underwent laboratory testing to determine the presence of infectious and systemic inflammatory diseases.

\section{Patient 1}

A 50-year-old woman with Down syndrome presented with a 5 -month history of ocular pain, proptosis, and periorbital swelling on the right orbit and low-grade fever. Contrast-enhanced CT (CECT) showed signs of pre- and postseptal cellulitis and scleritis of the right eye (Fig 1A). Infectious cellulitis was diagnosed and antibiotic therapy started. After a lack of improvement, an inflammatory etiology (IOID) was suspected and nonsteroidal anti-inflammatory drugs were initiated. Complete resolution of symptoms was subsequently achieved with systemic corticosteroids.

Eighteen months later, the same patient re-presented with similar complaints related to the left eye. Clinically, it was again thought to be infectious orbital cellulitis. On CECT, imaging findings on the left orbit were identical to the previous 
Table 1: Clinical data of patients with scleritis analyzed by CT and/or MR imaging

\begin{tabular}{|c|c|c|c|c|c|c|}
\hline Pt & $\begin{array}{l}\text { Duration of } \\
\text { Symptoms }^{\mathrm{a}}\end{array}$ & $\begin{array}{l}\text { US Performed, } \\
\text { Diagnosis }\end{array}$ & Clinical Diagnosis $^{b}$ & $\begin{array}{l}\text { Imaging } \\
\text { Modality }\end{array}$ & Systemic Disease & Final Diagnosis \\
\hline 1 & $5 \mathrm{mo}$ & No & Infectious cellulitis & $\mathrm{CT}$ & Down syndrome & IOID with scleritis \\
\hline 1 & $2 \mathrm{mo}$ & No & Infectious cellulitis & CT & Down syndrome & IOID with scleritis \\
\hline 2 & $3 \mathrm{mo}$ & Yes, disc edema & Optic neuritis & MRI & Not found & Bilateral idiopathic scleritis \\
\hline 3 & $2 \mathrm{mo}$ & No & Intraorbital mass & $\mathrm{CT}$ & Not found & Idiopathic scleritis \\
\hline 4 & $2 \mathrm{mo}$ & Yes, uveal mass & Choroidal melanoma & $\mathrm{CT}$ & Not found & Nodular idiopathic scleritis \\
\hline 5 & Acute & No & Infectious cellulitis & $\mathrm{CT}, \mathrm{MRI}$ & DRM; Colon carcinoma & $\begin{array}{l}\text { Infectious orbital disease } \\
\text { with panophthalmitis }\end{array}$ \\
\hline 6 & $6 \mathrm{mo}$ & No & Infectious cellulitis & CT, MRI & Down syndrome & IOID with sclerouveitis \\
\hline 7 & $1 \mathrm{mo}$ & Yes, scleritis & Scleritis or tumor & MRI & $\begin{array}{l}\text { Granulomatosis with } \\
\text { polyangiitis }\end{array}$ & $\begin{array}{l}\text { Autoimmune orbital } \\
\text { inflammation with scleritis }\end{array}$ \\
\hline 8 & $2 \mathrm{mo}$ & No & Uveitis & $\mathrm{CT}, \mathrm{MRI}$ & $J I A$ & Autoimmune sclerouveitis \\
\hline 9 & $3 w k$ & Yes, inconclusive & Optic pathway condition & $\mathrm{CT}, \mathrm{MRI}$ & None & Idiopathic scleritis \\
\hline 10 & $4 \mathrm{wk}$ & No & Optic pathway condition & MRI & None & Idiopathic scleritis \\
\hline
\end{tabular}

Note:-DRM indicates dermatomyositis; JIA, juvenile idiopathic arthritis; Pt, patient; US, ultrasonography.

${ }^{a}$ Duration of symptoms refers to the time elapsed between onset of symptoms of scleritis (pain, vision disturbances) and the time of imaging.

${ }^{\mathrm{b}}$ Diagnosis after ophthalmologic evaluation and ultrasound and before CT and/or MRI.
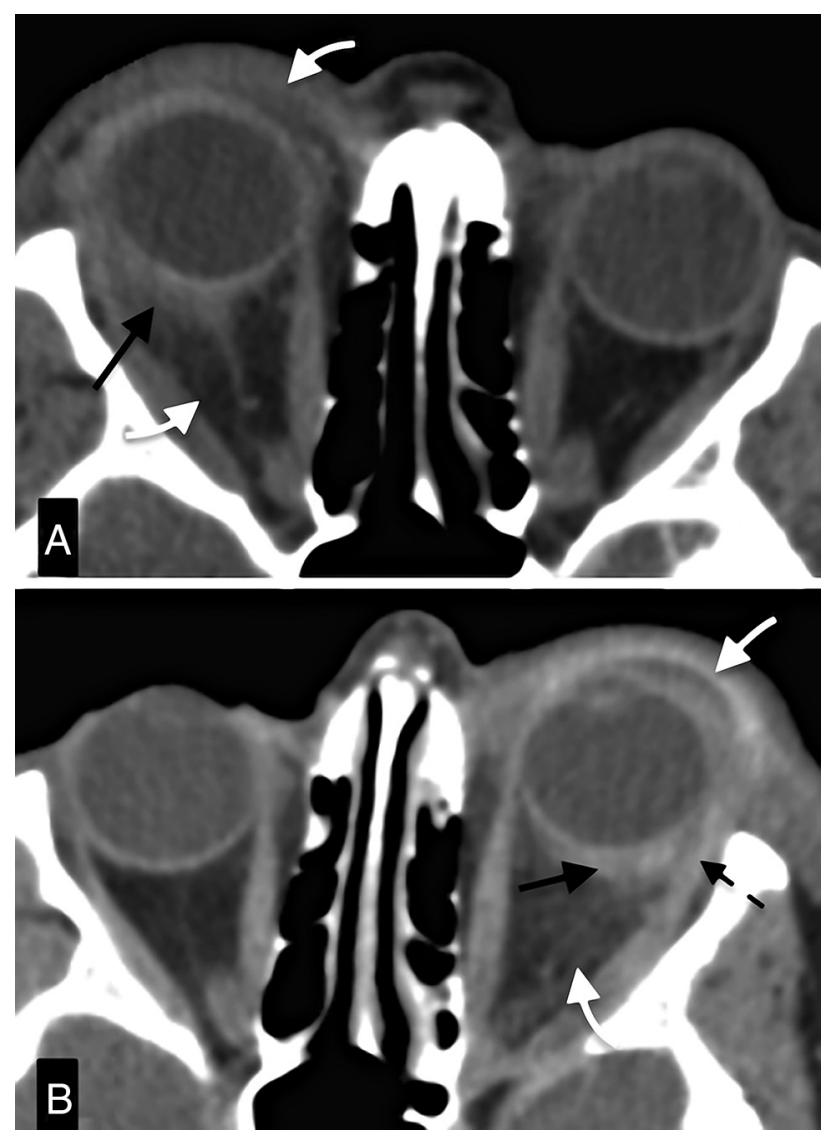

FIG 1. Asynchronous IOID with scleritis. A, CECT depicts outward, eccentric thickening and enhancement of the right globe wall with focal periscleral cellulitis (black arrow), compatible with posterior scleritis. There is associated pre- and postseptal cellulitis (white arrow) and proptosis. $B$, CECT 18 months after examination $(A)$ shows almost identical findings in the left orbit. Black and white arrows point to the scleritis and cellulitis, respectively. Notice the complete resolution of the alterations of the right orbit. Also, notice involvement of the tendon of the lateral rectus anteriorly (dashed arrow).

findings on the right side (Fig $1 B$ ). Because of the presence of scleritis and a history of pseudotumor, recurrent IOID (with scleritis) was diagnosed and corticosteroids started, with total recovery.
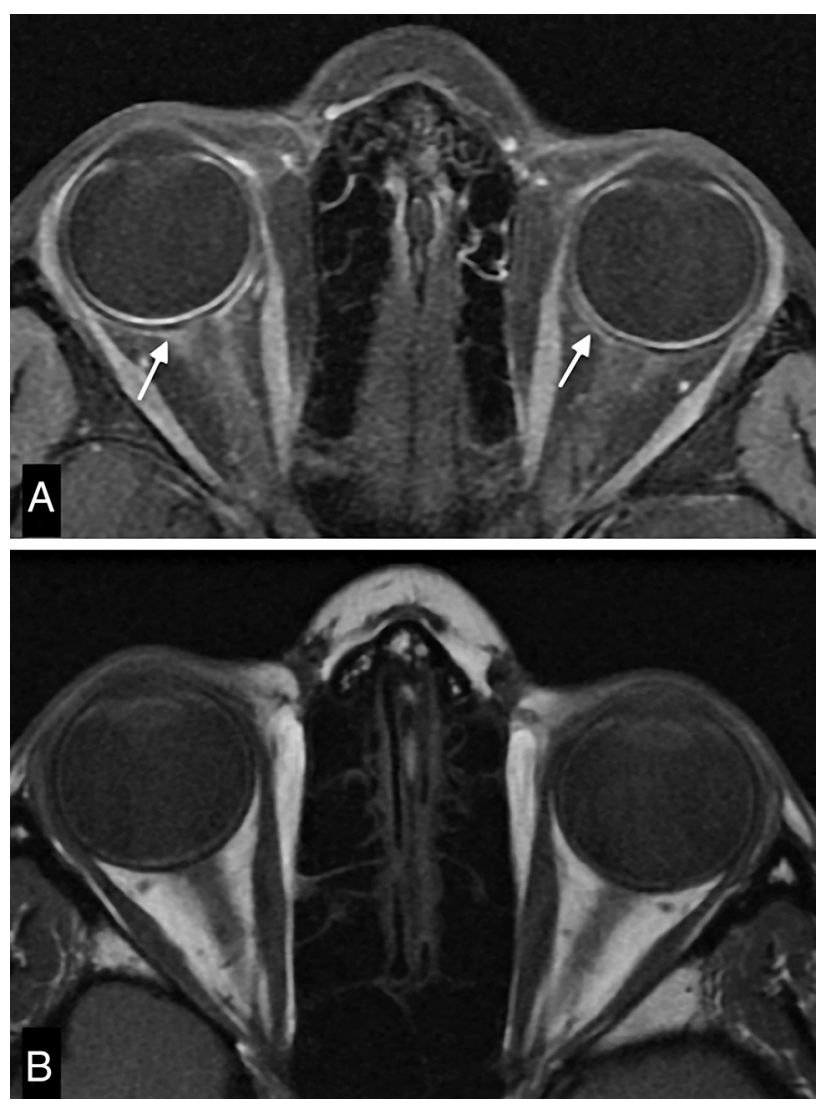

FIG 2. Bilateral inflammatory isolated scleritis: axial MR images. $A$, Post-Gd-DTPA T1-weighted image with fat saturation shows bilateral enhancement of the outer aspect of the sclera (white arrows) extending to the optic nerve sheath, depicting scleritis. There is also focal periscleral cellulitis. Notice the absence of ocular anomalies on the precontrast TIWI $(B)$.

\section{Patient 2}

An 11-year-old girl presented with 3 months of severe headaches that progressed to bilateral orbital pain and blurry vision. She had a history of recurrent peripheral articular pain. Ultrasonography revealed bilateral disc edema, and optic neuropathy was suspected. Gadolinium-enhanced MR imaging showed bilateral posterior scleritis (Fig $2 B$ ). In the absence of infectious parameters, an inflammatory idiopathic etiology was assumed and systemic cor- 
ticosteroids started, with improvement. Follow-up MR imaging showed no anomalies.

\section{Patient 3}

A 68-year-old woman presented with a 2-month history of proptosis and ocular pain on the left eye. Suspecting a retrobulbar mass, CECT was performed, depicting scleritis with periscleral cellulitis. Diagnosis of inflammatory idiopathic scleritis was made, and the patient was started on nonsteroidal anti-inflammatory drugs, with clinical improvement.

\section{Patient 4}

A 41-year-old woman was referred for enucleation by an ophthalmologist with the diagnosis of uveal melanoma. She had a 2-month history of vision loss and pain in the left eye. Fundoscopy and ultrasonography findings were compatible with a mass, but because of clinical uncertainty, including the presence of pain, a CECT was performed. It showed a scleral mass displacing the choroid anteriorly, with periscleral cellulitis (Fig 3). Given the cellulitis, sparing of the choroid, and the presence of pain, a diagnosis of inflammatory idiopathic nodular scleritis was suggested. Systemic corticosteroids were started, with complete resolution of symptoms, confirming the diagnosis.

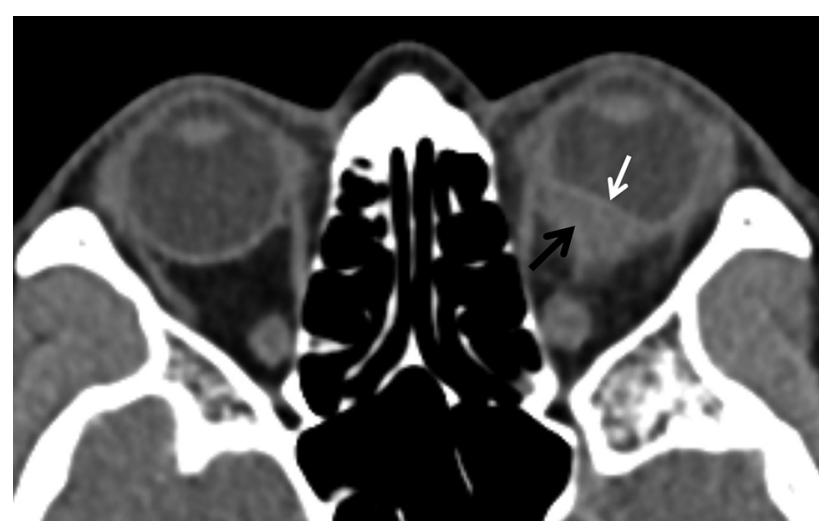

FIG 3. Nodular inflammatory scleritis mimicking uveal melanoma. CECT depicts a posterior globe wall mass (black arrow) deviating the choroid-retinal layer internally (white arrow), and hence, most probably arising from the sclera. Also notice the presence of slight periscleral cellulitis.
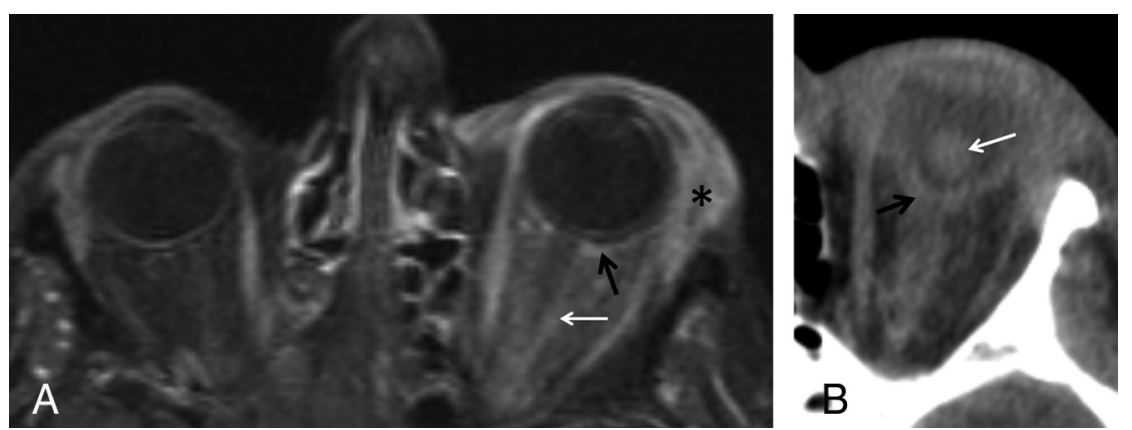

FIG 4. Infectious orbital process with scleritis followed by panophthalmitis. Post-Gd-DTPA axial T1-weighted spectral presaturation with inversion recovery image of the orbit $(A)$ depicts scleral enhancement (black arrow) and extensive pre- and postseptal cellulitis, with involvement of the optic nerve sheath (white arrow) and dacryoadenitis (asterisk). CECT performed 48 hours later (B) shows lens luxation (white arrow) and inward folding of the globe wall with volume loss (black arrow), depicting globe rupture.

\section{Patient 5}

A 66-year-old woman under immunosuppression for dermatomyositis presented with sudden onset of vision loss on the left, headache, proptosis, fever, and elevated infectious parameters. MR imaging depicted pre- and postseptal cellulitis, scleritis, and dacryoadenitis (Fig 4A). An orbital infection was suspected, and the patient was started on antibiotics. Despite treatment, infection progressed to panophthalmitis, and a subsequent CT showed globe rupture (Fig 4B). Cultures isolated Clostridium septicum.

\section{Patient 6}

A 56-year-old woman with Down syndrome complained of progressive right eyelid swelling and proptosis. Infectious cellulitis was suspected and the patient started on antibiotics, without improvement. An inflammatory idiopathic etiology was suspected and MR imaging performed, demonstrating pre- and postseptal cellulitis, scleritis, and uveitis, with choroidal and retinal detachments. Therapy with corticosteroids was initiated, with resolution of all ocular complaints.

\section{Patient 7}

An 80-year-old woman with granulomatosis with polyangiitis (Wegener) presented with a history of left ocular pain and 1 month of left eyelid swelling. Ultrasonography identified left posterior scleritis, but because of lack of improvement on nonsteroidal anti-inflammatory drugs, MR imaging was performed to exclude malignancy. It showed left scleritis (Fig 5), dacryoadenitis, and cellulitis. The findings were consistent with inflammatory autoimmune orbital disease, and corticosteroids were started. Resolution of symptoms was achieved after adding cyclophosphamide.

\section{Patient 8}

A 10-year-old boy with a history of idiopathic juvenile arthritis presented with ocular pain and diminished visual acuity of the left eye. Fundoscopy showed optic disc edema and signs of posterior uveitis, and orbital MR imaging depicted scleritis (Fig 6). Inflammatory autoimmune sclerouveitis was diagnosed, and corticosteroids and immunosuppressive medication were started, with clinical improvement.

\section{Patient 9}

A 47-year-old man presented with a 3-week history of headaches and blurred vision. Ultrasonography was inconclusive. CECT depicted posterior scleritis, confirmed by MR imaging (Fig 7). Assuming an inflammatory idiopathic etiology, nonsteroidal anti-inflammatory drugs were started, with complete resolution of symptoms.

\section{Patient 10}

An 8-year-old boy, with headaches for 4 weeks, presented with diminished visual acuity on the right eye. An optic neuropathy was suspected, and MR imaging 

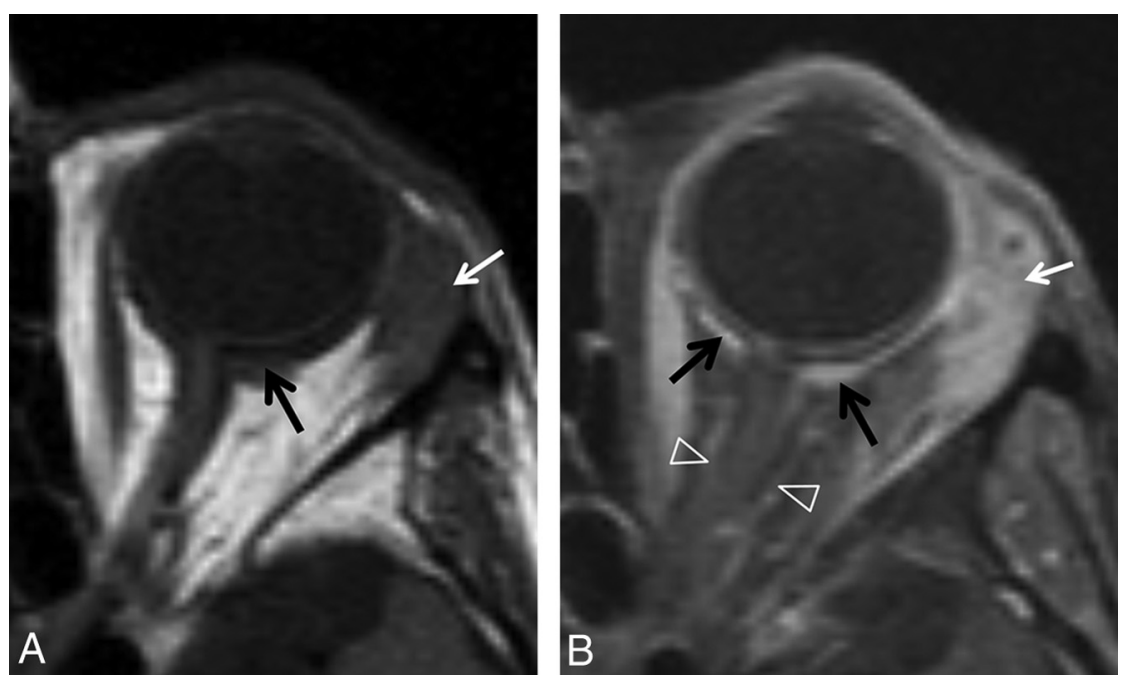

FIG 5. Orbital inflammation with scleritis in patient with granulomatosis with polyangiitis (Wegener). TIWI (A) shows eccentric focal thickening of the sclera (black arrow), with coexisting enlargement of the lacrimal gland (white arrow), both enhancing on the post-Gd-DTPA T1weighted spectral presaturation with inversion recovery image (B). Enhancement extends to the optic nerve sheath (open arrow heads). This illustrates posterior scleritis with optic perineuritis, cellulitis, and dacryoadenitis.

was performed, showing scleritis and focal periscleral cellulitis. An inflammatory idiopathic etiology was suspected and the patient started on corticosteroids and methotrexate, with visual recovery.

\section{DISCUSSION}

Although clinically scleritis is classified according to an anterior or posterior location and subdivided into diffuse or nodular (focal) forms, ${ }^{12,13}$ there has been no defined classification applied to imaging studies. Inflammation or infection of the sclera as observed on CT or MR imaging is generally referred to as "scleritis" independent of location or etiology. Anterior scleritis is the most common form, readily diagnosed on direct observation without the need for imaging. ${ }^{2,12}$ Posterior scleritis accounts for $2 \%-12 \%$ of cases and is widely underdiagnosed because of its rarity, variable clinical findings, and unfamiliarity with this condition of the general ophthalmologist and radiologist. ${ }^{2,6}$ The etiology of scleritis is most often inflammatory noninfectious, occurring either as an idiopathic condition (43\%) or as a manifestation of a systemic disease $(48 \%),{ }^{7,12,14}$ most often autoimmune. Rheumatoid arthritis and granulomatosis with polyangiitis are the most common underlying conditions. Idiopathic inflammatory scleritis might occur isolated or associated with extraocular anomalies and, we believe, as suggested by other authors, ${ }^{10,11,15-17}$ is part of the IOID/pseudotumor spectrum. We identified a systemic disease in 2 patients (granulomatosis with polyangiitis, juvenile idiopathic arthritis). In addition, 2 patients had Down syndrome, which to our knowledge has not been specifically associated with scleritis; both patients presented with extensive IOID. Infectious scleritis is rare, particularly in the absence of predisposing factors. ${ }^{3,4}$ In our series, 1 case was identified in an immunosuppressed patient.

The most distinctive clinical feature of scleritis is orbital pain, present in around $60 \%$ of patients. ${ }^{3}$ Orbital pain occurred in 7 of our 11 cases. From the remaining 4 cases, 3 presented with head- aches. Decreased vision has been described to occur in up to $31 \%$ of patients, 3\% developing permanent visual loss. ${ }^{1}$ We found visual symptoms in 6 cases, 5 of which resolved completely with treatment. Fever is a feature in infectious scleritis. Treatment depends on etiology and, excluding infectious causes, involves nonsteroidal anti-inflammatory drugs, corticosteroids, and immunosuppressive drugs. ${ }^{3,18}$

Correctly diagnosing scleritis is important given the potential for complications and the frequent association with systemic disease, of which scleritis might be the presenting manifestation. ${ }^{2,3,14}$ Because the posterior sclera cannot be directly visualized, imaging methods are needed to make or confirm the diagnosis in clinically challenging cases. Sonography is the most widely used imaging technique, but often fails to show pathognomonic findings and is of limited value in evaluating other intraorbital structures. ${ }^{2,19}$ Scleritis is frequently part of a more extensive inflammatory process, which might involve other orbital structures. In our series, sonography was performed by a specialized ophthalmologist in 4 patients and was diagnostic in 1 patient. Sonography was not performed in the remaining patients because scleritis was not clinically suspected.

MR imaging has excellent soft tissue contrast and the ability to image the entire orbit. On T2-weighted sequences, the posterior globe wall appears as a single hypointense ring, whereas on T1weighted sequences, the sclera can be individualized as the outer hypointense rim of the globe. A distinct hyperintense and enhancing rim can be seen internal to the sclera, corresponding to the choroid, and, at least partially, to the retina (Fig 2B).

Direct signs of scleritis on imaging are scleral enhancement, scleral thickening, and focal periscleral cellulitis. Our most consistent finding was scleral enhancement, present in $100 \%$ of contrast-enhanced examinations. Enhancement might involve the whole sclera (Fig $1 B$ ) or be preferentially peripheral (Fig 2), perhaps illustrating different degrees of inflammation, starting from the vascularized outer aspect of the sclera. It also might extend along the optic nerve sheath (Fig 5), representing optic perineuritis. Scleral enhancement is always abnormal and should not be confused with choroidal enhancement, a physiologic finding. Use of contrast is critical because scleral enhancement might be the only positive finding (Fig 2). ${ }^{5}$ On MR imaging, postcontrast T1WI should be acquired with fat saturation because the fat signal might mask scleral enhancement. Another sign of scleritis is scleral thickening, present in $83 \%$ of our patients and easily identified on MR imaging. On CT, though the globe layers cannot be separated, we found eccentric globe wall thickening and peripheral enhancement in all patients, making CT a useful tool in the diagnosis of scleritis. ${ }^{6,11,20}$ Periscleral cellulitis, described as the "ring sign" by Chaques et al, ${ }^{11}$ is also an important sign of scleritis, 

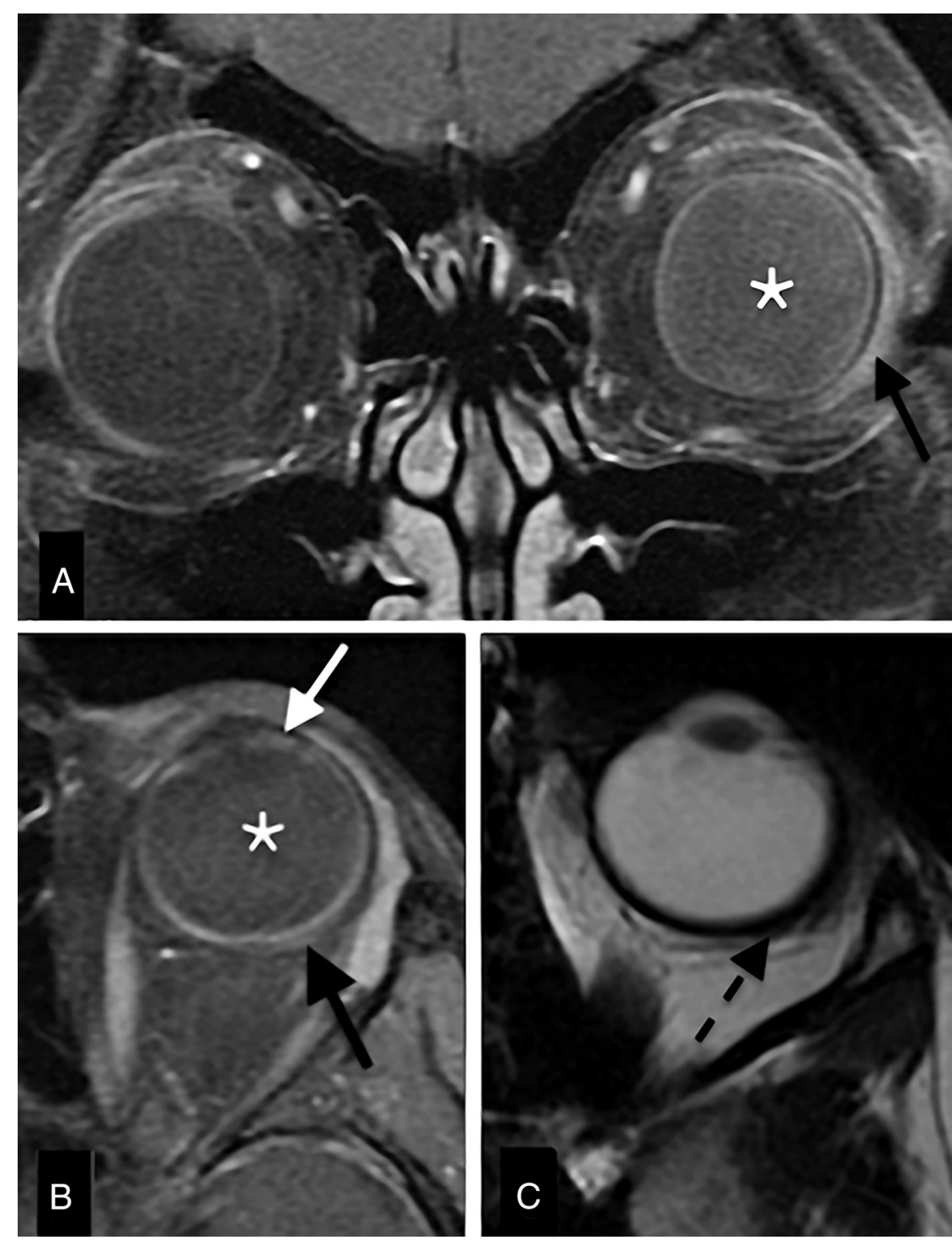

FIG 6. Scleritis with vitritis and uveitis. Enhanced coronal $(A)$ and axial (B) T1-weighted spectral presaturation with inversion recovery images and axial T2WI $(C)$ with a sclerouveitis. There is increased signal intensity of the vitreous on the left (vitritis; $A, B$, asterisk), with slight focal enhancement of the iris/cilliary body (uveitis; white arrow) and concurrent slight focal scleral outward thickening ( $C$, black dashed arrow) and enhancement $(B$, black solid arrow).

found in $42 \%$ of our patients. Imaging findings are summarized in Table 2.

Indirect signs of scleritis include retinal and choroidal detachment and effusion in the suprachoroidal or Tenon spaces. ${ }^{6}$ There might be associated uveitis, ${ }^{18,21}$ which can be differentiated from scleritis, particularly on MR imaging.

Scleritis also might be associated with extraocular orbital abnormalities, as seen in 5 of our cases, such as pre- and postseptal cellulitis, myositis, or dacryoadenitis. When scleritis occurs with anterior cellulitis, the clinical picture is often mistaken for infectious orbital cellulitis. Because extension of an orbital infection to the sclera is extremely rare in immunocompetent patients, coexistence of cellulitis and scleritis on imaging should raise suspicion of an inflammatory noninfectious etiology. ${ }^{3}$ Other clues pointing to an inflammatory etiology include sub- acute/chronic complaints and absence of fever, infectious parameters, or sinusitis.

The differential diagnosis of scleritis on imaging mainly is tumor, particularly with nodular scleritis, with published cases of globe tumors misdiagnosed as scleritis ${ }^{14}$ and scleritis mimicking a choroidal mass. ${ }^{8,9}$ In the diffuse form of scleritis, posterior uveitis, diffuse melanoma, and lymphoma are the most relevant differential diagnoses. CT and MR imaging have proved useful in distinguishing these entities. ${ }^{20}$ In challenging cases, a therapeutic trial (with nonsteroidal anti-inflammatory drugs or steroids $)^{15}$ and/or histopathologic confirmation might be warranted because misdiagnoses of scleritis have been described to lead to inadvertent enucleations. ${ }^{9}$ In the presented case with melanoma as the presumptive diagnosis (patient 4), imaging and treatment with corticosteroids led to resolution of the mass, making a biopsy unnecessary. Clues to the inflammatory (versus neoplastic) nature of the process include the presence of pain, cellulitis as seen on imaging, and the specific scleral location, though some intraocular tumors might also involve the sclera. Additional MR sequences, such as DWI, might be of benefit, ${ }^{22}$ particularly in lymphoma, which often demonstrates restricted diffusion. ${ }^{23}$ Neither DWI nor other advanced imaging sequences were routinely performed in our patients.

Although we present a small group of patients, they illustrate a wide range of direct and indirect imaging findings in scleritis. CT and MR imaging provided useful information and influenced clinical decision-making. MR imaging is the most useful examination in the diagnosis of scleritis, ${ }^{5,9}$ differentiating the sclera from the other ocular layers. In our experience and as ascertained by previous studies, ${ }^{6,11,20}$ CT is also able to diagnose scleritis, showing eccentric outward globe wall thickening and enhancement, frequently associated with periscleral cellulitis. Both techniques are more informative than ultrasonography for assessment of extraocular extension of disease.

We suggest that in cases when clinical evaluation and ultrasonography do not suffice in the diagnosis of scleritis, or when associated ailments are suspected, MR imaging be performed. When MR imaging is not available, or when there are contraindications, CT can be of diagnostic value.

The results of our study are limited by its retrospective nature, the small number of patients, and the lack of a standardized pro- 

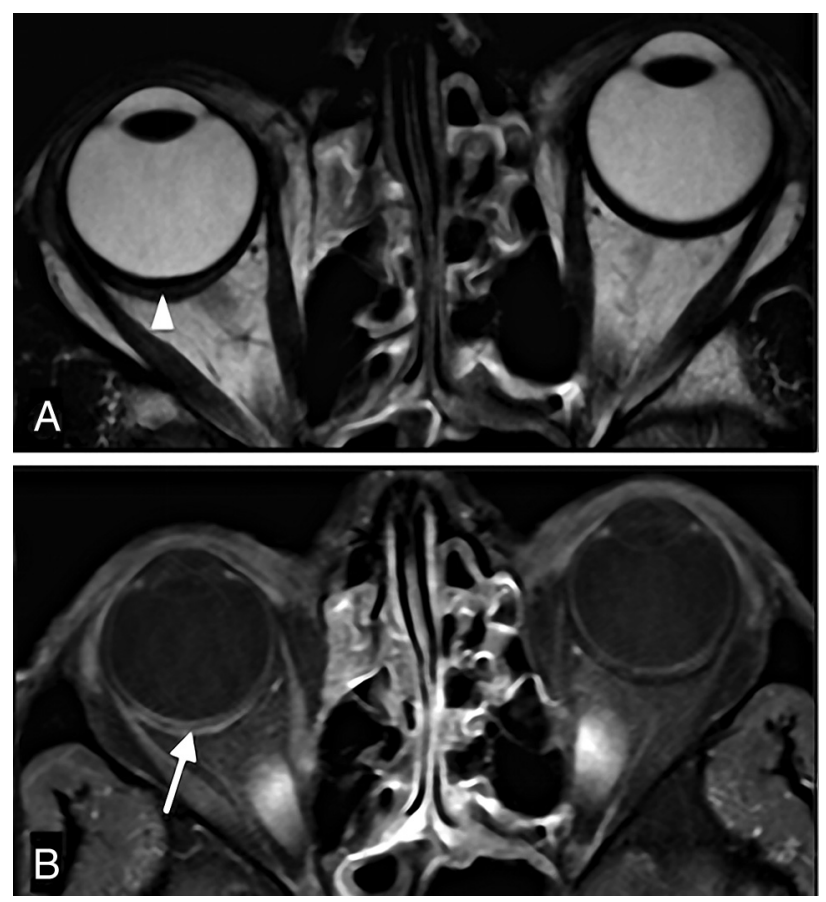

FIG 7. Isolated inflammatory scleritis: orbital MR imaging. T2-weighted (A) and Gd-DTPA enhanced Th-weighted spectral presaturation with inversion recovery $(B)$ images depict scleral thickening and enhancement ( $B$, white arrow). There is a linear hyperintense (fluid) collection between the sclera and the choroid/retina, representing a suprachoroidal effusion (A, white arrowhead).

Table 2: Imaging findings in 11 cases with scleritis on CT $(n=8)$ and MRI $(n=8)$

\begin{tabular}{lll}
\hline $\begin{array}{c}\text { Imaging } \\
\text { Modality }\end{array}$ & \multicolumn{1}{c}{ Imaging Finding } & No. (\%) \\
\hline CT & Eccentric enhancement of the globe wall & $8(100)$ \\
& Eccentric thickening of the sclerouveal rim & $8(100)$ \\
& Periscleral cellulitis & $6(75)$ \\
& Pre/postseptal cellulitis & $4(50)$ \\
& Nodular scleral tickening & $1(13)$ \\
MR & Scleral enhancement & $8(100)$ \\
& Scleral thickening & $6(75)$ \\
& Focal periscleral cellulitis & $4(50)$ \\
& Pre/postseptal cellulitis & $2(25)$ \\
& Scleral thinning & $1(13)$ \\
& Dacryoadenitis & $1(13)$ \\
& Uveitis & $2(25)$ \\
& Suprachoroidal effusion & $1(13)$ \\
Retinal detachment & $1(13)$ \\
& Choroidal detachment & $1(13)$ \\
\hline
\end{tabular}

tocol. Despite this, we believe that this article contributes to the current knowledge on imaging signs of scleritis on CT and MR imaging. To the best of our knowledge, this is the largest series of MR imaging in scleritis analyzed to date.

\section{CONCLUSIONS}

Scleritis is an underdiagnosed, treatable condition, almost invariably of inflammatory noninfectious etiology-idiopathic, in the spectrum of IOID, or in the context of a systemic disease. Imaging findings on CT and particularly on MR imaging are quite distinct and include scleral enhancement, scleral thickening, and focal periscleral cellulitis. To the radiologist, familiarization with these findings is important to recognize this diagnosis.
Disclosures: Martine Jager-UNRELATED: Travel/Accommodations/Meeting Expenses Unrelated to Activities Listed: Costs made for travel as visiting professor have been repaid by universities.

\section{REFERENCES}

1. McCluskey PJ, Watson PG, Lightman S, et al. Posterior scleritis: clinical features, systemic associations, and outcome in a large series of patients. Ophthalmology 1999;106:2380-86 CrossRef Medline

2. Benson WE. Posterior scleritis. Surv Ophthalmol 1988;32:297-316 CrossRef Medline

3. Biber J, Schwam B, Raizman M. Scleritis. In: Krachmer J, Mannis M, Holland E, eds. Cornea. 3rd ed. St Louis: Mosby; 2011

4. Ho YF, Yeh LK, Tan HY, et al. Infectious scleritis in Taiwan-a 10-year review in a tertiary-care hospital. Cornea 2014;33:838-43 CrossRef Medline

5. Cordero-Coma M, García-Morán A, Yilmaz T, et al. Adjunctive globe magnetic resonance imaging in the diagnosis of posterior scleritis. Can J Ophthalmol 2011;46:329-32 CrossRef Medline

6. Biswas J, Mittal S, Ganesh SK, et al. Posterior scleritis: clinical profile and imaging characteristics. Indian J Ophthalmol 1998;46:195-202 Medline

7. Mafee MF, Karimi A, Shah J, et al. Anatomy and pathology of the eye: role of MR imaging and CT. Radiol Clin N Am 2006;44:135-57 CrossRef

8. Osman Saatci A, Saatci I, Kocak N, et al. Magnetic resonance imaging characteristics of posterior scleritis mimicking choroidal mass. Eur J Radiol 2001;39:88-91 CrossRef Medline

9. Maggioni F, Ruffatti S, Viaro F, et al. A case of posterior scleritis: differential diagnosis of ocular pain. J Headache Pain 2007;8:123-26 CrossRef Medline

10. Johnson MH, DeFilipp GJ, Zimmerman RA, et al. Scleral inflammatory disease. AJNR Am J Neuroradiol 1987;8:861-65 Medline

11. Chaques VJ, Lam S, Tessler HH, et al. Computed tomography and magnetic resonance imaging in the diagnosis of posterior scleritis. Ann Ophthalmol 1993;25:89-94 Medline

12. Okhravi N, Odufuwa B, McCluskey P, et al. Scleritis. Surv Ophthalmol 2005;50:351-63 CrossRef Medline

13. Watson PG, Hayreh SS. Scleritis and episcleritis. Br J Ophthalmol 1976;60:163-91 CrossRef Medline

14. Kafkala C, Daoud YJ, Paredes I, et al. Masquerade scleritis. Ocul Immunol Inflamm 2005;13:479-82 CrossRef Medline

15. Gordon LK. Orbital inflammatory disease: a diagnostic and therapeutic challenge. Eye (Lond) 2006;20:1196-206 CrossRef Medline

16. Uehara F, Ohba N. Diagnostic imaging in patients with orbital cellulitis and inflammatory pseudotumor. Int Ophthalmol Clin 2002; 42:133-42 CrossRef Medline

17. Ding ZX, Lip G, Chong V. Idiopathic orbital pseudotumour. Clin Radiol 2011;66:886-92 CrossRef Medline

18. Wieringa WG, Wieringa JE, ten Dam-van Loon NH, et al. Visual outcome, treatment results, and prognostic factors in patients with scleritis. Ophthalmology 2013;120:379-86 CrossRef Medline

19. Benson WE, Shields JA, Tasman W, et al. Posterior scleritis. A cause of diagnostic confusion. Arch Ophthalmol 1979;97:1482-86 CrossRef Medline

20. Dorey SE, Clark BJ, Christopoulos VA, et al. Orbital lymphoma misdiagnosed as scleritis. Ophthalmology 2002;109:2347-50 CrossRef Medline

21. Cheung CM, Chee SP. Posterior scleritis in children: clinical features and treatment. Ophthalmology 2012;119:59-65 CrossRef Medline

22. Kapur R, Sepahdari AR, Mafee MF, et al. MR imaging of orbital inflammatory syndrome, orbital cellulitis, and orbital lymphoid lesions: the role of diffusion-weighted imaging. AJNR Am J Neuroradiol 2009;30:64-70 Medline

23. Razek AA, Elkhamary S, Mousa A. Differentiation between benign and malignant orbital tumors at 3-T diffusion MR-imaging. Neuroradiology 2011;53:517-22 CrossRef Medline 